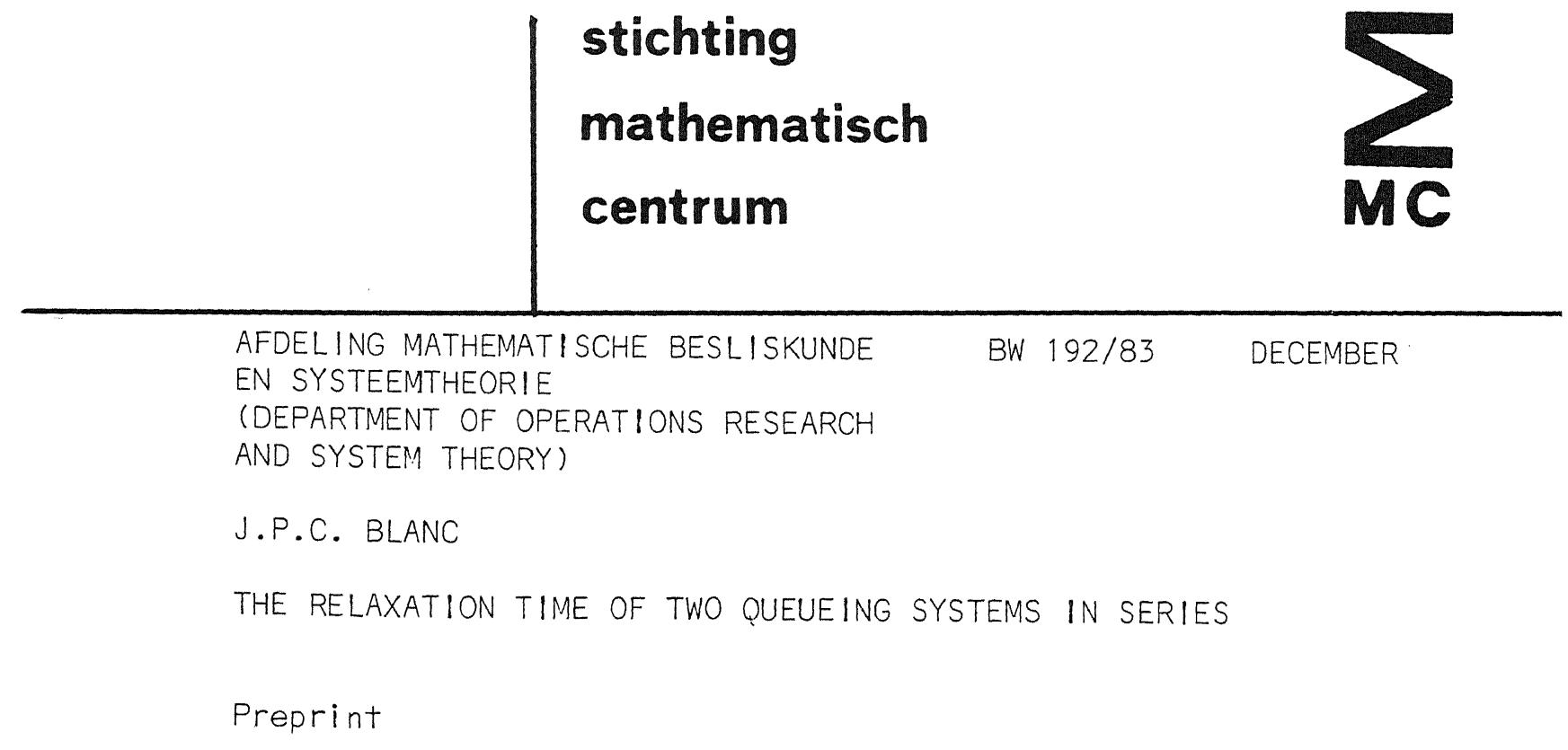


Printed at the Mathematical Centre, Kruislaan 413, Amsterdam, The Netherlands.

The Mathematical Centre, founded 11 February 1946, is a non-profit institution for the promotion of pure and applied mathematics and computer science. It is sponsored by the Netherlands Government through the Netherlands Organization for the Advancement of Pure Research (Z.W.O.).

1980 Mathematics subject classification: 90B22, 60K25

Copyright (C) 1983, Mathematisch Centrum, Amsterdam 


\title{
The relaxation time of two queueing systems in series $\dagger$
}

\author{
by
}

J.P.C. Blanc

\begin{abstract}
This paper deals with the time-dependent behaviour of two queueing systems in series, the simplest example of a Jackson network. The Laplace transform of the probability $p_{0}(t)$ that the tandem system is empty at time $t$ is obtained by reducing the functional equation for the generating function of the joint queue length distribution to a Riemann-Hilbert boundary value problem. From this Laplace transform the relaxation time of $p_{0}(t)$ is determined for all cases, and the first term of the asymptotic expansion of $p_{0}(t)-p_{0}(\infty)$ as $t \rightarrow \infty$ is found in the ergodic and in the null recurrent cases.
\end{abstract}

KEY WORDS \& PHRASES: Tandem queueing system, relaxation time, asymptotic expansion, Riemann-Hilbert boundary value problem, conformal mapping. 


\section{Introduction}

This paper is devoted to the study of the asymptotic behaviour as $t$ (time) $\rightarrow \infty$ of the distribution of the number of jobs in a tandem queueing system with a Poisson arrival stream, two exponential single server stations, independent service times at the two stations and no blocking. The investigation of the asymptotic behaviour of the distribution of the number of jobs in a queueing system is important for obtaining insight into the rate of convergence to the stationary queue length distribution (if this exists). Concerning the asymptotic behaviour of systems with a single queue many results have been obtained, see e.g. [3], [5], [13], [14]. For these systems the concept of relaxation time (or its inverse the decay parameter) has been introduced as a measure for the rate of convergence of the time-dependent distribution to its limiting value as $t \rightarrow \infty$. However, little is known about the relaxation times of queueing networks. In [10] the time-dependent behaviour of a two-node cyclic network has been studied with the aid of a diffusion approximation. In [8] an iterative scheme is derived for the solution of the timedependent Kolmogorov equations for Gordon-Newell type networks, but this method does not provide much insight into the relaxation time for this kind of networks. Recently an analytical method has been developed for the study of queueing systems with two queues, cf. [6],[7],[4],[1],[2]. This method consists of the solution of the functional equation for the generating function of the distribution of the number of jobs in the system with the aid of the theory of boundary value problems for regular functions and by using the conformal mapping of the unit disk onto a given domain. It is applicable not only in the stationary, but also in the time-dependent case, see [1],[2],[4]. In this paper the above mentioned method will be used to obtain the transform of the time-dependent distribution of the number of jobs in a tandem queueing system. From that transform the first term (apart from the limiting value) of the asymptotic expansion (as $t \rightarrow \infty$ ) of the probability that the system is empty will be derived. For the tandem queueing model we define

$\alpha$ : the mean interarrival time for jobs at the first node,

$\beta_{j}: \quad$ the mean service time at node $j, j=1,2$,

$a_{j:=} \beta_{j} / \alpha, \quad$ the traffic intensity at node $j, j=1,2$.

The service times of a job at the two nodes are assumed to be independent. For this model it is well known, cf.[9], that the system is stable iff $a_{1}<1$ and $a_{2}<1$, and that the stationary probability that there are $k_{1}$ jobs at node 1 and $k_{2}$ jobs at node 2 is equal to

$$
\left(1-a_{1}\right) a_{1}{ }^{k_{1}}\left(1-a_{2}\right) a_{2}{ }^{k_{2}}, \quad k_{1}, k_{2}=0,1,2, \ldots
$$

To put our results for the tandem model into perspective we repeat the asymptotic results for the $M / M / 1$ model. For $j=1,2$, the probability $p_{j}(t)$ that an $M / M / 1$ system with mean interarrival time $\alpha$ and mean service time $\beta_{j}$ is empty at time $t, t>0$, given $p_{j}(0)=1$, is determined by, cf.[3], $\S$ I.4.4,

$$
\int_{0}^{\infty} e^{-\rho t} p_{j}(t) d t=\frac{\beta_{j} \nu_{j}(\rho)}{1-\nu_{j}(\rho)},
$$

here

$$
v_{j}(\rho):=\frac{1}{2 a_{j}}\left\{1+\beta_{j} \rho+a_{j}-\sqrt{\left[1+\beta_{j} \rho+a_{j}\right]^{2}-4 a_{j}}\right\} .
$$

The larger singularity (branch point) of $\nu_{j}(\rho)$ is

$$
\rho_{j}:=-\left[1-\sqrt{a_{j}}\right]^{2} / \beta_{j}, \quad j=1,2 .
$$

With the aid of the saddle point method, cf.[14],[3], $\S 1.4 .4$, it follows from (2) that for $j=1,2$, as $t \rightarrow \infty$,

$$
p_{j}(t)=\left[1-a_{j}\right]^{+}+\frac{a_{j}^{\frac{1}{4}}}{\left(1-\sqrt{a_{j}}\right)^{2}} \frac{e^{-\left(1-\sqrt{\left.a_{j}\right)^{2} t / \beta_{j}}\right.}}{2 \sqrt{\pi}\left(t / \beta_{j}\right)^{3 / 2}}-\left[1+O\left(\frac{1}{t}\right)\right], \text { if } a_{j} \neq 1,
$$




$$
=\left(\pi t / \beta_{j}\right)^{-1 / 2}\left[1+0\left(\frac{1}{t}\right)\right], \text { if } a_{j}=1 .
$$

The relaxation time of these $M / M / 1$ systems is defined to be $T_{j}:=-1 / \rho_{j}, j=1,2$, cf.(4).

Our results concerning the relaxation time for the probability that the tandem queueing system is empty are the following: in the ergodic case $a_{1}<1, a_{2}<1$, it is equal to the maximum of the relaxation times $T_{1}, T_{2}$ of the service systems at the two nodes when considered separately as $M / M / 1$ queues; if $a_{1}>1, a_{2}<1\left(a_{1}<1, a_{2}>1\right)$, it is equal to the relaxation time $T_{1}\left(T_{2}\right)$ of the transient node; if $a_{1}>1$, $a_{2}>1$, it is less than or equal to the minimum of $T_{1}, T_{2}$.

In the sections $2,3,4,5$ the transform of the time-dependent joint distribution of the number of jobs at the two nodes of the tandem system will be determined. In section 6 the relaxation time will be discussed for the ergodic as well as for the non-ergodic case. Section 7 is devoted to the asymptotic expansion of the probability that the tandem system is empty. Finally, in an appendix the conformal mapping which has to be introduced for the solution of the functional equation will be discussed.

\section{The functional equation and its kernel}

Let $N_{j}(t), j=1,2$, be the number of jobs present at the $j^{\text {th }}$ station at time $t \geqslant 0$ and define for $\operatorname{Re} \rho>0,\left|z_{1}\right| \leqslant 1,\left|z_{2}\right| \leqslant 1$,

$$
\Omega\left(\rho ; z_{1}, z_{2}\right):=\int_{0}^{\infty} e^{-\rho t} E\left\{z_{1}^{\underline{N}_{1}(t)} z_{2}^{-N_{2}^{(t)}} \mid \underline{N}_{1}(0)=\underline{N}_{2}(0)=0\right\} d t,
$$

i.e. the discussion is restricted to the case that the tandem system is empty at time $t=0$. In a standard way it can be derived from the Kolmogorov equations for the tandem queueing system that the transform $\Omega\left(\rho ; z_{1}, z_{2}\right)$ must satisfy the following functional equation: for $\operatorname{Re} \rho>0,\left|z_{1}\right| \leqslant 1,\left|z_{2}\right| \leqslant 1$,

$$
\begin{gathered}
{\left[\alpha \rho+1-z_{1}+\frac{1}{a_{1} z_{1}}\left(z_{1}-z_{2}\right)+\frac{1}{a_{2} z_{2}}\left(z_{2}-1\right)\right] \Omega\left(\rho ; z_{1}, z_{2}\right)=} \\
=\alpha+\frac{1}{a_{1} z_{1}}\left(z_{1}-z_{2}\right) \Omega\left(\rho ; 0, z_{2}\right)+\frac{1}{a_{2} z_{2}}\left(z_{2}-1\right) \Omega\left(\rho ; z_{1}, 0\right) .
\end{gathered}
$$

An important role in the analysis of this functional equation is played by the kernel,

$$
K\left(\rho ; z_{1}, z_{2}\right):=\alpha \rho+1-z_{1}+\frac{1}{a_{1} z_{1}}\left(z_{1}-z_{2}\right)+\frac{1}{a_{2} z_{2}}\left(z_{2}-1\right),
$$

because zeros of this kernel in the region $\operatorname{Re} \rho>0,\left|z_{1}\right| \leqslant 1,\left|z_{2}\right| \leqslant 1$, where the transform $\Omega\left(\rho ; z_{1}, z_{2}\right)$ is known to be finite, lead to the relation, cf.(7),

$$
\frac{1}{a_{1} z_{1}}\left(z_{2}-z_{1}\right) \Omega\left(\rho ; 0, z_{2}\right)+\frac{1}{a_{2} z_{2}}\left(1-z_{2}\right) \Omega\left(\rho ; z_{1}, 0\right)=\alpha
$$

The existence of such zeros of the kernel can easily be proved with Rouché's theorem; e.g. for $\rho, z_{1}$ fixed, $\operatorname{Re} \rho>0,\left|z_{1}\right|=1$, there exists a unique $z_{2}$ in the unit disk for which the kernel $K\left(\rho ; z_{1}, z_{2}\right)$ vanishes. In the next section we shall investigate the zeros of the kernel $K\left(\rho ; z_{1}, z_{2}\right)$ in more detail. This section we conclude with the derivation of some special values of $\Omega\left(\rho ; z_{1}, z_{2}\right)$ which will be needed later.

For $z_{2}=1$ equation (7) becomes the functional equation for an $M / M / 1$ system (station 1 ), for which the solution reads, cf. [3], § I.4.4, for $\operatorname{Re} \rho>0,\left|z_{1}\right| \leqslant 1$, cf.(2),

$$
\Omega\left(\rho ; z_{1}, 1\right)=\frac{\beta_{1} \nu_{1}(\rho)}{1-\nu_{1}(\rho)} \frac{1}{1-a_{1} z_{1} \nu_{1}(\rho)} .
$$

Note that the marginal distribution of $\underline{N}_{1}(t)$ obviously has relaxation time $T_{1}$. Further, by using that $K\left(\rho ; \nu_{2}(\rho), \nu_{2}(\rho)\right)=0, K\left(\rho ; \nu_{2}(\rho), a_{1} / a_{2}\right)=0$, cf. $(8),(2)$, it follows that for $a_{1} \leqslant a_{2}$, 


$$
\Omega\left(\rho ; 0, a_{1} / a_{2}\right)=\frac{\beta_{2} \nu_{2}(\rho)}{1-\nu_{2}(\rho)}
$$

In the case $a_{1}=a_{2}$ this relation coincides with (10) for $z_{1}=0$. But by differentiation of both sides of (7) it follows in this case that

$$
\left.\frac{\partial}{\partial z_{2}} \Omega\left(\rho ; 0, z_{2}\right)\right|_{z_{2}=1}=\frac{\beta_{1} \nu_{1}(\rho)}{1-\nu_{1}(\rho)} \frac{a_{1} \nu_{1}^{2}(\rho)}{1-a_{1} \nu_{1}^{2}(\rho)} .
$$

\section{The zeros of the kernel}

From (8) it is seen that all zeros of $K\left(\rho ; z_{1}, z_{2}\right)$ can be described by the two valued function

$$
\begin{gathered}
z_{2}\left(z_{1}\right)=\frac{1}{2} a_{1} z_{1}\left\{A(\rho)-z_{1} \pm \sqrt{\left[A(\rho)-z_{1}\right]^{2}-\frac{4}{a_{1} a_{2} z_{1}}}\right\}, \\
A(\rho):=\alpha \rho+1+\frac{1}{a_{1}}+\frac{1}{a_{2}} .
\end{gathered}
$$

In this section we shall study the properties of the function $z_{2}\left(z_{1}\right)$ without paying attention to the conditions $\operatorname{Re} \rho>0,\left|z_{1}\right| \leqslant 1,\left|z_{2}\left(z_{1}\right)\right| \leqslant 1$, but with the restriction to real values of $\rho$.

Lemma 1. The function $z_{2}\left(z_{1}\right)$ has four branch points; one of them is $z_{1}=0$. The other three branch points are real and positive if

$$
\rho \geqslant \rho_{0}:=\left[-1-\frac{1}{a_{1}}-\frac{1}{a_{2}}+3\left(\frac{1}{a_{1} a_{2}}\right)^{\frac{1}{3}}\right] / \alpha .
$$

Proof. That $z_{1}=0$ is a branch point of $z_{2}\left(z_{1}\right)$ is clear from (13). The other branch points are the zeros of the function

$$
\left[A(\rho)-z_{1}\right]^{2}-\frac{4}{a_{1} a_{2} z_{1}} .
$$

As $z_{1} \downarrow 0$ this function tends to $-\infty$. For $\rho>0$ it is positive at $z_{1}=1$ and negative at $z_{1}=A(\rho)>1$, while it tends to $+\infty$ as $z_{1} \rightarrow \infty$. This proves that the three zeros of (16) are real and positive for $\rho>0$. Clearly the three zeros of (16) are still real and positive when $\rho$ decreases to negative values until the largest value of $\rho$ for which the function of $z_{1}$ in (16) has a double zero. A simple calculation shows that this value is $\rho_{0}$ as defined in (15).

In the rest of this paper the values of $\rho$ are restricted to $\rho \geqslant \rho_{0}$. Further we shall denote by $v(\rho)$ the smallest positive branch point of $z_{2}\left(z_{1}\right)$. Note that $0<v(\rho)<1$ for $\rho>0$ by the proof of lemma 1 .

Lemma 2. For $z_{1} \in[0, v(\rho)]$ the values of $z_{2}\left(z_{1}\right)$ lie on the contour $Z(\rho)$ defined by

$$
\left|z_{2}\right|^{2}=\frac{a_{1}}{2 a_{2}}\left\{A(\rho)-\sqrt{A^{2}(\rho)-\frac{8}{a_{1}} \operatorname{Re} z_{2}}\right\},\left|z_{2}\right|^{2}<\frac{a_{1}}{a_{2}} v(\rho) .
$$

Proof. For $z_{1} \in[0, v(\rho)]$ the function of $z_{1}$ in (16) is negative, so that it follows from (13) that

$$
\left|z_{2}\left(z_{1}\right)\right|^{2}=\frac{a_{1}}{a_{2}} z_{1}, \quad \operatorname{Re} z_{2}\left(z_{1}\right)=\frac{1}{2} a_{1} z_{1}\left\{A(\rho)-z_{1}\right\} .
$$

These relations lead to the equation (17), where the minus sign in front of the square root has to be taken because $z_{2}(0)=0$ and $z_{2}(v(\rho))=\left[a_{1} v(\rho) / a_{2}\right]^{\frac{1}{2}}$ have to lie on the contour.

Lemma 3. The branch point $v(\rho)$ of the function $z_{2}\left(z_{1}\right)$ is a strictly decreasing function of $\rho$ for $\rho \geqslant \rho_{0}$, van- 
ishing as $\rho \rightarrow \infty$.

Proof. Because $v(\rho)$ is a zero of (16) it follows by differentiation that

$$
\frac{d}{d \rho} v(\rho)=-\alpha[v(\rho)]^{\frac{3}{2}}\left\{\left(a_{1} a_{2}\right)^{-\frac{1}{2}}-[v(\rho)]^{\frac{3}{2}}\right\}^{-1}
$$

It is readily verified that as $\rho \rightarrow \infty$ one of the zeros of (16) vanishes, while the other two zeros tend to infinity. Since $v(\rho)$ is defined to be the smallest zero of (16) it vanishes as $\rho \rightarrow \infty$. Further, $v(\rho)=\left(a_{1} a_{2}\right)^{-\frac{1}{3}}$ if and only if $\rho=\rho_{0}$, so that by continuity $0<v(\rho)<\left(a_{1} a_{2}\right)^{-\frac{1}{3}}$ for $\rho>\rho_{0}$. Hence, by (19) the derivative of $v(\rho)$ is negative for $\rho>\rho_{0}$.

Next define $x(\rho):=\sqrt{a_{1} v(\rho) / a_{2}}$; this is one of the two points at which the contour $Z(\rho)$ crosses the real axis, cf. lemma 2. From (17) it follows that $x=x(\rho)$ is the smallest positive root of the equation

$$
\frac{a_{2}}{a_{1}} x^{3}+\frac{2}{a_{2}}=A(\rho) x .
$$

Lemma 4. For $\rho>\rho_{0}$ the contour $Z(\rho)$ possesses a tangent at every point. The contour $Z\left(\rho_{0}\right)$ possesses a tangent at every point except at $x\left(\rho_{0}\right)$; here the limits of the tangents at $z_{2}$ as $z_{2} \rightarrow x\left(\rho_{0}\right)$ in the upper half plane and in the lower half plane form a corner point with inner angle 2 arctan $\sqrt{3}=\frac{2}{3} \pi$.

Proof. Because the function $z_{2}\left(z_{1}\right)$, cf.(13), is analytic (conformal) at every point except at its four branch points, and because the contour $Z(\rho)$ is the image of a real interval under the mapping $z_{1} \rightarrow z_{2}\left(z_{1}\right)$, the contour $Z(\rho)$ possesses a tangent at least at every point not corresponding to a branch point of $z_{2}\left(z_{1}\right)$. The points $z_{1}=0$ (for every $\rho$ ) and $z_{1}=v(\rho)$ (for $\rho>\rho_{0}$ ) are first order branch points which implies that at $z_{2}=0$ and at $z_{2}=x(\rho), \rho>\rho_{0}$, the contour $Z(\rho)$ has a tangent perpendicular to the real axis. For $\rho=\rho_{0}$ it follows from (17) that for $z_{2} \in Z\left(\rho_{0}\right)$,

$$
\operatorname{Im} z_{2}= \pm \sqrt{3}\left\{x\left(\rho_{0}\right)-\operatorname{Re} z_{2}\right\}+o\left(x\left(\rho_{0}\right)-\operatorname{Re} z_{2}\right), \quad \operatorname{Re} z_{2} \uparrow x\left(\rho_{0}\right) ;
$$

this proves the last statement of the lemma.

The interior of the contour $Z(\rho)$ defined by (17) will be denoted by $Z^{+}(\rho)$, the exterior of this contour by $Z^{-}(\rho), \rho \geqslant \rho_{0}$.

Lemma 5. If $a_{1}<a_{2}^{2}$ then $1 \in Z^{-}(\rho)$ for $\rho \geqslant \rho_{0}$; if $a_{1} \geqslant a_{2}^{2}$ then

$$
1 \in Z^{-}(\rho), \text { iff } \alpha \rho>\frac{\left(1-a_{2}\right)\left(a_{1}-a_{2}\right)}{a_{1} a_{2}} \text {. }
$$

If $a_{2}<a_{1}^{2}$ then $a_{1} / a_{2} \in Z^{-}(\rho)$ for $\rho \geqslant \rho_{0}$; if $a_{2} \geqslant a_{1}^{2}$ then

$$
a_{1} / a_{2} \in Z^{-}(\rho), \text { iff } \alpha \rho>\frac{\left(1-a_{1}\right)\left(a_{2}-a_{1}\right)}{a_{1} a_{2}} \text {. }
$$

Proof. As a consequence of lemma 3 the point $x(\rho)$ on $Z(\rho)$ is a strictly decreasing function of $\rho$ for $\rho \geqslant \rho_{0}$, so that it attains its maximum value for $\rho=\rho_{0}$, while $x\left(\rho_{0}\right)=\left(a_{1} / a_{2}^{2}\right)^{\frac{1}{3}}$. Hence, $1 \in Z^{-}(\rho)$ for $\rho \geqslant \rho_{0}$, iff $1>x\left(\rho_{0}\right)$, which is equivalent to $a_{1}<a_{2}^{2}$. If $1 \leqslant x\left(\rho_{0}\right)$, i.e. in the case $a_{1} \geqslant a_{2}^{2}$, then $1 \in Z(\rho)$ iff $1=x(\rho)$, or equivalently by (20), iff $A(\rho)=a_{2} / a_{1}+2 / a_{2}$, cf.(14). By the monotonicity of $x(\rho)$ we thus 
have $1 \in Z^{-}(\rho)$ iff $A(\rho)>a_{2} / a_{1}+2 / a_{2}$. The second part of the lemma can be proved similarly.

\section{The boundary value problem and its index}

In this section a Riemann-Hilbert boundary value problem will be formulated for the function $\Omega\left(\rho ; 0, z_{2}\right)$, and the index of this Riemann-Hilbert problem will be determined with the aid of the results of the preceding section. Throughout this section $\rho$ is real, $\rho \geqslant \rho_{0}$.

Lemma 6. For $\rho>0$ the function $\Omega\left(\rho ; 0, z_{2}\right)$ is regular in the domain $Z^{+}(\rho)$ and satisfies for $z_{2} \in Z(\rho)$ the boundary condition

$$
\operatorname{Re}\left[i \frac{a_{2} z_{2}}{a_{1} z_{1}} \quad \frac{z_{2}-z_{1}}{1-z_{2}} \Omega\left(\rho ; 0, z_{2}\right)\right]=\operatorname{Re}\left[\frac{i \beta_{2} z_{2}}{1-z_{2}}\right] .
$$

Proof. By definition $K\left(\rho ; z_{1}, z_{2}\left(z_{1}\right)\right)=0$, so that (9) holds for $z_{2}=z_{2}\left(z_{1}\right),\left|z_{1}\right| \leqslant 1,\left|z_{2}\left(z_{1}\right)\right| \leqslant 1$. In the preceding section we saw that $v(\rho)<1$ for $\rho>0$. Further, if $x(\rho)<1$, i.e. $1 \in Z^{-}(\rho)$, cf. lemma 5 , then $Z(\rho)$ lies entirely inside the unit circle, because $x(\rho)$ is the point with the largest absolute value on $Z(\rho)$, cf. (18). Hence, if $x(\rho)<1$ then $\Omega\left(\rho ; 0, z_{2}\right)$ is regular in $Z^{+}(\rho)$, and (21) follows from (9) by taking $z_{1} \in[0, v(\rho)]$ where $\Omega\left(\rho ; z_{1}, 0\right)$ is real. If $x(\rho)>1$ then $\Omega\left(\rho ; 0, z_{2}\left(z_{1}\right)\right)$ can be continued analytically over the interval $[0, v(\rho)]$ by means of the relation (9), because $\Omega\left(\rho ; z_{1}, 0\right)$ is regular on this interval. Hence, (the analytic continuation of $\Omega\left(\rho ; 0, z_{2}\right)$ is finite at $z_{2}=x(\rho)$. Because $\Omega\left(\rho ; 0, z_{2}\right)$ has a power series expansion at $z_{2}=0$ with positive coefficients, this implies that $\Omega\left(\rho ; 0, z_{2}\right)$ is regular for $\left|z_{2}\right|<x(\rho)$, and hence in $Z^{+}(\rho)$.

In lemma 6 an inhomogeneous Riemann-Hilbert problem, cf.[11], $\S 40,41$, has been formulated for the function $\Omega\left(\rho ; 0, z_{2}\right)$. In order to apply the solution method described in [11] the boundary condition (21) on $Z(\rho)$ has to be transformed conformally to the unit circle. Therefore we introduce $\gamma(\rho ; z)$ as a conformal mapping of the region $|z| \leqslant 1$ onto $Z^{+}(\rho) \cup Z(\rho)$. Because the real axis is an axis of symmetry of the contour $Z(\rho)$, cf.(17), $\gamma(\rho: z)$ may be chosen such that

$$
\gamma(\rho ; \bar{z})=\overline{\gamma(\rho ; z)}, \quad \gamma(\rho ;-1)=0, \quad \gamma(\rho ; 1)=x(\rho) .
$$

The conformal mapping $\gamma(\rho ; z)$ is determined by (22) up to the value of $\gamma(\rho ; 0)$, which is restricted to the real interval $(0, x(\rho))$. See [1], § I.6, for relevant references on conformal mappings. In the appendix an expression will be derived for $\gamma_{0}\left(\rho ; z_{2}\right)$, the inverse conformal mapping of $\gamma(\rho ; z)$. For $z_{2}=\gamma(\rho ; z),|z|=1$, and $K\left(\rho ; z_{1}, z_{2}\right)=0$, it follows from (18) that

$$
z_{1}=\frac{a_{2}}{a_{1}}|\gamma(\rho ; z)|^{2}=\frac{a_{2}}{a_{1}} \gamma(\rho ; z) \gamma(\rho ; \bar{z})=\frac{a_{2}}{a_{1}} \gamma(\rho ; z) \gamma\left(\rho ; \frac{1}{z}\right)
$$

With the above the boundary condition (21) can be rewritten as: for $|z|=1$,

$$
\begin{gathered}
\operatorname{Re}\left[i \frac{\gamma(\rho ; z)}{\gamma(\rho ; 1 / z)} \frac{1-a_{2} \gamma(\rho ; 1 / z) / a_{1}}{1-\gamma(\rho ; z)} \Omega(\rho ; 0, \gamma(\rho ; z))\right]= \\
=\frac{1}{2} i \beta_{2}\left[\frac{\gamma(\rho ; z)}{1-\gamma(\rho ; z)}-\frac{\gamma(\rho ; 1 / z)}{1-\gamma(\rho ; 1 / z)}\right]=\frac{1}{2} i \beta_{2} \frac{\gamma(\rho ; z)-\gamma(\rho ; 1 / z)}{|1-\gamma(\rho ; z)|^{2}} .
\end{gathered}
$$

The index $\kappa$ of this Riemann-Hilbert problem is defined, cf.[11], $\S 41$, to be the increment of the function

$$
\frac{1}{2 \pi i} \log \left[\left(\frac{\gamma(\rho ; 1 / z)}{\gamma(\rho ; z)}\right)^{2} \frac{1-a_{2} \gamma(\rho ; z) / a_{1}}{1-a_{2} \gamma(\rho ; 1 / z) / a_{1}} \frac{1-\gamma(\rho ; z)}{1-\gamma(\rho ; 1 / z)}\right],
$$

when $z$ traverses the unit circle once.

Lemma 7. The index of the Riemann-Hilbert problem (24) is given by (cf. lemma 5): 


$$
\begin{aligned}
& \kappa=-2, \text { if } 1 \in Z^{-}(\rho) \text { and } \frac{a_{1}}{a_{2}} \in Z^{-}(\rho), \\
& \kappa=0, \quad \text { if } 1 \in Z^{+}(\rho), \frac{a_{1}}{a_{2}} \in Z^{-}(\rho), \text { or if } 1 \in Z^{-}(\rho), \frac{a_{1}}{a_{2}} \in Z^{+}(\rho), \\
& \kappa=+2, \text { if } 1 \in Z^{+}(\rho), \frac{a_{1}}{a_{2}} \in Z^{+}(\rho) .
\end{aligned}
$$

Proof. Because $\gamma(\rho ;-1)=0$ and $\gamma(\rho ; 1)>0$, cf. (22), the function

$$
\frac{\gamma(\rho ; 1 / z)}{\gamma(\rho ; z)}=\left[\frac{\gamma(\rho ; 1 / z)}{|\gamma(\rho ; 1 / z)|}\right]^{2}
$$

traverses the unit circle once in negative direction, so that - for every $\rho \geqslant \rho_{0}$ - the index of this function is equal to -2 . Clearly, the index of the function

$$
\left[1-a_{2} \gamma(\rho: z) / a_{1}\right] /\left[1-a_{2} \gamma(\rho ; 1 / z) / a_{1}\right],
$$

is equal to 0 if $a_{1} / a_{2} \in Z^{-}(\rho)$ and equal to +2 if $a_{1} / a_{2} \in Z^{+}(\rho)$, and similarly for the last factor in (25). Because the indices of the different factors in (25) may be added, this proves the assertion.

Note that the case $\kappa=0$ does not occur when $a_{1}=a_{2}$. Further it is seen from lemma 5 that the case $\kappa=+2$ only occurs if $a_{1}>a_{2}^{2}$ and $a_{2}>a_{1}^{2}$, i.e. $a_{1}^{2}<a_{2}<\sqrt{a_{1}}<1$, and only for negative values of $\rho$.

\section{The solution of the Riemann-Hilbert problem}

In this section it will be shown that the Riemann-Hilbert problem (24) possesses a solution in the case $\kappa=-2$. Further, the transform $\Omega(\rho ; 0,0), \rho>0$, and its continuation to $\rho \geqslant \rho_{0}$, will be determined.

Throughout this section, $\rho$ is real, $\rho \geqslant \rho_{0}$, and $C:=\{z ;|z|=1\}$.

Lemma 8. For $\rho$ such that $1 \in Z^{-}(\rho)$ and $a_{1} / a_{2} \in Z^{-}(\rho)$,

$$
\Omega(\rho ; 0,0)=\left[\gamma^{\prime}(\rho ;-1)\right]^{-2} \beta_{2} I_{C}(\rho),
$$

with

$$
\begin{aligned}
& I_{C}(\rho):=\frac{1}{2 \pi i} \int_{C} \frac{\gamma(\rho ; u)-\gamma(\rho ; 1 / u)}{u+1}|\psi(\rho ; u)|^{2} \frac{d u}{u}, \\
& \psi(\rho ; u):=\gamma(\rho ; u)\left[(u+1)\{1-\gamma(\rho ; u)\}\left\{1-a_{2} \gamma(\rho ; u) / a_{1}\right\}\right]^{-1} .
\end{aligned}
$$

Proof. For the values of $\rho$ under consideration the index $\kappa$ of the Riemann-Hilbert problem (24) is equal to -2 , cf. lemma 7 . Let

$$
\begin{aligned}
& F(\rho ; z):=\frac{\gamma(\rho ; z)}{\gamma(\rho ; 1 / z)} \frac{1-a_{2} \gamma(\rho ; 1 / z) / a_{1}}{1-\gamma(\rho ; z)}, \\
& f(\rho ; z):=\frac{1}{2} \beta_{2} i \frac{\gamma(\rho ; z)-\gamma(\rho ; 1 / z)}{|1-\gamma(\rho ; z)|^{2}} ;
\end{aligned}
$$

then by [11], $\S 40,41$, the Riemann-Hilbert problem (24) with index $\kappa=-2$ has a unique solution given by : for $|z|<1$,

$$
\Omega(\rho ; 0, \gamma(\rho ; z))=\frac{X(\rho ; z)}{\pi i} \int_{C} \frac{f(\rho ; u)}{i F(\rho ; u) X(\rho ; u)} \frac{d u}{u-z}
$$

with 


$$
X(\rho ; z):=\exp \left[\frac{1}{2 \pi i} \int_{C} \log \left\{u^{2} \frac{F(\rho ; 1 / u)}{F(\rho ; u)}\right\} \frac{d u}{u-z}\right],|z| \leqslant 1
$$

if the following condition is satisfied:

$$
\int_{C} \frac{f(\rho ; u) d u}{F(\rho ; u) X(\rho ; u)}=0 .
$$

In order to determine the function $X(\rho ; z)$ we note that for $|z|<1$,

$$
\begin{gathered}
\int_{C} \log \left\{u^{2} \frac{F(\rho ; 1 / u)}{F(\rho ; u)}\right\} \frac{d u}{u-z}=\int_{C}\left[2 \log \left\{\frac{\gamma(\rho ; 1 / u)}{1+1 / u}\right\}-2 \log \left\{\frac{\gamma(\rho ; u)}{1+u}\right\}+\right. \\
\left.+\log \left\{1-\frac{a_{2}}{a_{1}} \gamma(\rho ; u)\right\}-\log \left\{1-\frac{a_{2}}{a_{1}} \gamma\left(\rho ; \frac{1}{u}\right)\right\}+\log \{1-\gamma(\rho ; u)\}-\log \left\{1-\gamma\left(\rho ; \frac{1}{u}\right)\right\}\right] \frac{d u}{u-z} .
\end{gathered}
$$

Because $\gamma(\rho ;-1)=0$ and $\gamma^{\prime}(\rho ;-1) \neq 0$ exists (the latter can be proved by application of Kellogg's theorem, cf. [12], theorem IX.7, [1], theorem II.8.2), because $\gamma(\rho ; u)$ is regular for $|u|<1$, and because $1 \in Z^{-}(\rho), a_{1} / a_{2} \in Z^{-}(\rho)$, all these logarithmic terms are regular functions either inside or outside the unit circle. Hence, each term can be determined with the residue theorem. This leads to: for $|z| \leqslant 1$,

$$
X(\rho ; z)=\left[\gamma(\rho ; 0) \frac{z+1}{\gamma(\rho ; z)}\right]^{2} \frac{1-a_{2} \gamma(\rho ; z) / a_{1}}{1-a_{2} \gamma(\rho ; 0) / a_{1}} \frac{1-\gamma(\rho ; z)}{1-\gamma(\rho ; 0)} .
$$

With (28) and (32) condition (31) becomes, cf.(27),

$$
\int_{C}\left[\gamma(\rho ; u)-\gamma\left(\rho ; \frac{1}{u}\right)\right]|\psi(\rho ; u)|^{2} \frac{d u}{u}=0
$$

which is readily seen to be satisfied. Finally, $\Omega(\rho ; 0,0)$ follows by taking $z=-1$ in $(29)$ and by substituting (28) and (32) in (29).

Because for all values of the parameters $a_{1}$ and $a_{2}$ the index of the Riemann-Hilbert problem (24) becomes -2 as $\rho \rightarrow \infty$, cf. lemma 3 , the transform $\Omega(\rho ; 0,0)$ is determined for every $a_{1}, a_{2}$ in the region $\operatorname{Re} \rho>0$ by analytic continuation from (26). Explicit expressions for $\Omega(\rho ; 0,0)$ for real $\rho$ such that $1 \in Z^{+}(\rho)$ and/or $a_{1} / a_{2} \in Z^{+}(\rho)$ can be obtained by solving the Riemann-Hilbert problems with index 0 and 2 and by using the relations (10) and/or (11) to determine the constants in the solution of the Riemann-Hilbert problem with $\kappa \geqslant 0$, cf.[11]. The same expressions can be obtained by noting that e.g. as $\alpha \rho \downarrow\left(1-a_{2}\right)\left(a_{1}-a_{2}\right) / a_{1} a_{2}$ then $\gamma(\rho ; 1)=x(\rho) \uparrow 1$, so that the integral in (26) becomes singular, and hence by taking into account the residues of the singularities. It turns out that for $\alpha \rho<\left(1-a_{2}\right)\left(a_{1}-a_{2}\right) / a_{1} a_{2}$, cf. lemma 5, the following term must be added in the expression (26) for $\Omega(\rho ; 0,0)$ :

$$
\left[\gamma^{\prime}(\rho ;-1)\right]^{-2} \frac{\beta_{2}}{\gamma^{\prime}\left(\rho ; \gamma_{0}(\rho ; 1)\right)} \frac{1-\gamma_{0}(\rho ; 1)}{\left[1+\gamma_{0}(\rho ; 1)\right]^{3}} \frac{a_{1}}{a_{1}-a_{2}} \frac{a_{1} \gamma\left(\rho ; 1 / \gamma_{0}(\rho ; 1)\right)}{a_{1}-a_{2} \gamma\left(\rho ; 1 / \gamma_{0}(\rho ; 1)\right)} .
$$

By using the fact that $\gamma_{0}\left(\rho ; z_{2}\right)$ is the inverse of $\gamma(\rho ; z)$, cf. (22), and by using the results (A.3) and (A.8) from the appendix, the additional term (33) can be rewritten as, cf.(3),

$$
\Delta_{1}(\rho):=\frac{\beta_{1} \nu_{1}(\rho)}{1-\nu_{1}(\rho)} \frac{a_{1}+a_{2}^{2}-a_{2}\left[1+a_{1}+\beta_{1} \rho\right]}{a_{1}-a_{2}} .
$$

Analogously, the following term must be added in the expression $(26)$ for $\Omega(\rho ; 0,0)$ when $a_{1} / a_{2} \in Z^{+}(\rho)$, cf. lemma 5 ,

$$
\Delta_{2}(\rho):=\frac{\beta_{2} \nu_{2}(\rho)}{1-\nu_{2}(\rho)} \frac{a_{2}+a_{1}^{2}-a_{1}\left[1+a_{2}+\beta_{2} \rho\right]}{a_{2}-a_{1}}
$$


In the case $a_{1}=a_{2}<1$ it takes some more effort to obtain the additional term for $\rho<0$, but straightforward calculations lead to:

$$
\Delta_{0}(\rho):=\frac{\beta_{1} \nu_{1}(\rho)}{1-\nu_{1}(\rho)}\left[2\left(1-a_{1}\right)+\beta_{1} \rho \frac{a_{1} \nu_{1}^{2}(\rho)}{1-a_{1} \nu_{1}^{2}(\rho)}\right]
$$

These results are summarized below ( see also lemma 5):

Lemma 9. The transform $\Omega(\rho ; 0,0)$ is given by (26) if $1 \in Z^{-}(\rho)$ and $a_{1} / a_{2} \in Z^{-}(\rho)$, and it is given by

$$
\begin{array}{cl}
{\left[\gamma^{\prime}(\rho ;-1)\right]^{-2} \beta_{2} I_{C}(\rho)+\Delta_{1}(\rho),} & \text { if } 1 \in Z^{+}(\rho), \frac{a_{1}}{a_{2}} \in Z^{-}(\rho), \\
{\left[\gamma^{\prime}(\rho ;-1)\right]^{-2} \beta_{2} I_{C}(\rho)+\Delta_{2}(\rho),} & \text { if } 1 \in Z^{-}(\rho), \frac{a_{1}}{a_{2}} \in Z^{+}(\rho), \\
{\left[\gamma^{\prime}(\rho ;-1)\right]^{-2} \beta_{2} I_{C}(\rho)+\Delta_{1}(\rho)+\Delta_{2}(\rho),} & \text { if } 1 \in Z^{+}(\rho), \frac{a_{1}}{a_{2}} \in Z^{+}(\rho), a_{1} \neq a_{2}, \\
{\left[\gamma^{\prime}(\rho ;-1)\right]^{-2} \beta_{2} I_{C}(\rho)+\Delta_{0}(\rho),} & \text { if } a_{1}=a_{2}<1, \rho<0 .
\end{array}
$$

\section{The relaxation time}

The relaxation time of the probability that a queueing system is empty is determined by that singularity of its Laplace transform which has the largest real part, in the ergodic case apart from a pole at the origin, cf.[3],§ III.7.3. Therefore, the abscissa of convergence has to be determined of the Laplace transform, cf.(1),

$$
\begin{aligned}
& \Omega^{\star}(\rho):=\Omega(\rho ; 0,0)-\rho^{-1}\left[1-a_{1}\right]^{+}\left[1-a_{2}\right]^{+}= \\
& =\int_{0}^{\infty} e^{-\rho t}\left[\operatorname{Pr}\left\{\underline{N}_{1}(t)=\underline{N}_{2}(t)=0\right\}-\operatorname{Pr}\left\{\underline{N}_{1}(\infty)=\underline{N}_{2}(\infty)=0\right\}\right] d t .
\end{aligned}
$$

Theorem 1. The relaxation time of the probability that the tandem queueing system is empty is given by

$$
\begin{aligned}
& T_{1}:=\frac{\beta_{1}}{\left(1-\sqrt{a_{1}}\right)^{2}}, \quad \text { if } a_{1} \geqslant a_{2} \text { and } a_{1} \geqslant a_{2}^{2}, \\
& T_{2}:=\frac{\beta_{2}}{\left(1-\sqrt{a_{2}}\right)^{2}}, \quad \text { if } a_{2} \geqslant a_{1} \text { and } a_{2} \geqslant a_{1}^{2}, \\
& T_{0}:=\frac{\alpha a_{1} a_{2}}{a_{1} a_{2}+a_{1}+a_{2}-3\left(a_{1} a_{2}\right)^{2 / 3}}, \text { otherwise. }
\end{aligned}
$$

Proof. In each expression for the Laplace transform $\Omega(\rho ; 0,0)$ in lemma 8 and 9 the term $I_{C}(\rho)$ appears. This integral is defined only for real $\rho, \rho>\rho_{0}$, but because the conformal mapping $\gamma(\rho ; u)$ can be continued analytically into the half plane $\operatorname{Re} \rho>\rho_{0}$, cf. the appendix, also $I_{C}(\rho)$ can be continued into this half plane. At points in this half plane where the integrand of $I_{C}(\rho)$ has a pole the principle value of $I_{C}(\rho)$ exists, except in the case $a_{1}=a_{2}<1, \rho=0$, at $\mathrm{u}=1$. Because in the latter case this pole of $I_{C}(\rho)$ at $\rho=0$ has been subtracted in (41), it follows that in general the absissa of convergence of $\Omega \star(\rho)$ is at least $\rho_{0}$, cf.(15). The only factor of $\Delta_{j}(\rho), j=0,1,2$, cf. (34),(35),(36), which can have an abscissa of convergence larger than $\rho_{0}$ is $\nu_{j}(\rho), j=1,2$. From (3) it is seen that the abscissa of convergence of $\nu_{j}(\rho)$ is the branch point $\rho_{j}, j=1,2$, cf.(4). It is not difficult to prove that for every $a_{1}, a_{2}$,

$$
\rho_{j} \geqslant \rho_{0}, \quad\left[\rho_{j}=\rho_{0} \text { iff } a_{j}=a_{3-j}^{2}\right], \quad j=1,2 .
$$

Hence, the abscissa of convergence of $\Omega \star(\rho)$ is $\rho_{j}$ whenever $\nu_{j}(\rho)$ occurs in an expression for $\Omega \star(\rho)$, cf. 
lemma 5,9, $j=1,2$. Only in the case $a_{1}<1, a_{2}<1$, it can happen that both $\nu_{1}(\rho)$ and $\nu_{2}(\rho)$ occur, cf. lemma 5,(39). Then the abcissa of convergence of $\Omega(\rho)$ is determined by the larger of $\rho_{1}$ and $\rho_{2}$. It is readily found that when $a_{1}<1, a_{2}<1$,

$$
\rho_{1}>\rho_{2} \text { iff } a_{1}>a_{2}, \quad \rho_{1}=\rho_{2} \text { iff } a_{1}=a_{2} .
$$

Finally, when the abscissa of convergence of $\Omega \star(\rho)$ is $\rho_{j}, j=0,1,2$, then by definition the relaxation time is equal to $T_{j}=-\rho_{j}^{-1}$.

Note that $T_{j}, j=1,2$, is equal to the relaxation time of a single $M / M / 1$ queueing system with arrival rate $\alpha^{-1}$ and mean service time $\beta_{j}$. Hence, theorem 1 states that in the ergodic case $a_{1}<1, a_{2}<1$ the relaxation time of two exponential service stations in series is the maximum, cf. (43), of the relaxation times of each of the stations separately. This leads to the conjecture that the relaxation time of an ergodic system of $n$ stations in series or even of feedforward Jackson networks is the maximum of the relaxation times of each of the components.

For the transient case theorem 1 states that the relaxation time of the tandem system is equal to or less than $\left(T_{0}\right)$ the minimum of the relaxation times of those components which do not possess a marginal stationary distribution. This is in agreement with the inequalities for $t \geqslant 0$,

$$
\operatorname{Pr}\left\{\underline{N}_{1}(t)=\underline{N}_{2}(t)=0\right\} \leqslant \operatorname{Pr}\left\{\underline{N}_{j}(t)=0\right\}, j=1,2 ;
$$

because for every real $\sigma$ for which the righthand side multiplied by $\exp (\sigma t)$ vanishes as $t \rightarrow \infty$ also the lefthand side multiplied by exp $(\sigma t)$ has to vanish as $t \rightarrow \infty$.

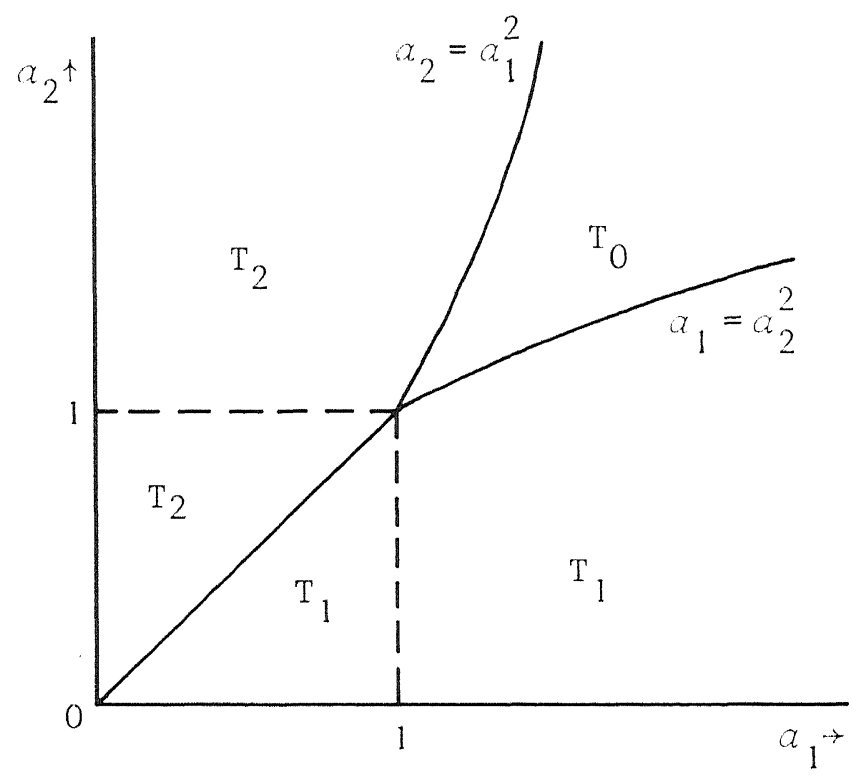

Figure: The relaxation time of two queues in series as function of the parameters $a_{1}, a_{2}$. 


\section{Asymptotic expansions}

This section is concerned with a refinement of theorem 1, i.e. in the ergodic case the second term in the asymptotic expansion of $p_{0}(t):=\operatorname{Pr}\left\{\underline{N}_{1}(t)=\underline{N}_{2}(t)=0\right\}$ as $t \rightarrow \infty$ will be determined, and in the null-recurrent case, when the relaxation time is infinite, the first term of the above expansion will be derived.

Theorem 2. In the case $a_{2}<a_{1}<1$, as $t \rightarrow \infty$,

$$
p_{0}(t)=\left(1-a_{1}\right)\left(1-a_{2}\right)+\frac{1}{2 \sqrt{\pi}} \frac{a_{1}^{\frac{1}{4}}}{\left(1-\sqrt{a_{1}}\right)^{2}} \frac{\left(\sqrt{a_{1}}-a_{2}\right)^{2}}{a_{1}-a_{2}} \frac{e^{-t / T_{1}}}{\left(t / \beta_{1}\right)^{\frac{3}{2}}}\left[1+O\left(\frac{1}{t}\right)\right] ;
$$

in the case $a_{1}<a_{2}<1$, as $t \rightarrow \infty$,

$$
p_{0}(t)=\left(1-a_{1}\right)\left(1-a_{2}\right)+\frac{1}{2 \sqrt{\pi}} \frac{a_{2}^{\frac{1}{4}}}{\left(1-\sqrt{a_{2}}\right)^{2}} \frac{\left(\sqrt{a_{2}}-a_{1}\right)^{2}}{a_{2}-a_{1}} \frac{e^{-t / T_{2}}}{\left(t / \beta_{2}\right)^{\frac{3}{2}}}\left[1+O\left(\frac{1}{t}\right)\right] ;
$$

in the case $a_{1}=a_{2}<1$, as $t \rightarrow \infty$,

$$
p_{0}(t)=\left(1-a_{1}\right)^{2}+\frac{1}{2 \sqrt{\pi}} a_{1}^{\frac{1}{4}}\left(1-\sqrt{a_{1}}\right) \frac{e^{-t / T_{1}}}{\left(t / \beta_{1}\right)^{1 / 2}}\left[1+O\left(\frac{1}{t}\right)\right] .
$$

Proof. In the case $a_{2}<a_{1}<1$ the asymptotic behaviour of $p_{0}(t)$ as $t \rightarrow \infty$ is determined by the branch point of $\Omega \star(\rho)$ at $\rho=\rho_{1}$, cf. theorem 1. Because in this case $\rho_{1}>\rho_{3}$ and $\rho_{1}>\rho_{2}$, the only singular component of $\Omega \star(\rho)$ at $\rho=\rho_{1}$ is the function $\nu_{1}(\rho)$, cf. lemma 9. Hence, in this case the asymptotic expansion of $p_{0}(t)$ follows readily from the results of the analysis of the $M / M / 1$ system, cf. (2),(5),(4),(34). The case $a_{1}<a_{2}<1$ can be treated similarly. In the case $a_{1}=a_{2}<1$ the asymptotic behaviour of $p_{0}(t)$ is also determined by the singularity of $\Omega \star(\rho)$ at $\rho=\rho_{1}$, but in this case there is not only a branch point at $\rho=\rho_{1}$, but also a pole, because $\nu_{1}\left(\rho_{1}\right)=a_{1}^{-1 / 2}$, cf. (3),(4),(36). But by the saddle point method the expansion of $p_{0}(t)$ is obtained in a similar way as in [14], only deleting an integration by parts.

In the case $a_{1}=a_{2}<1$ the convergence of $p_{0}(t)$ to its limiting value as $t \rightarrow \infty$ seems to be slower than that of $p_{1}(t)$ for the same traffic intensity $a_{1}$, cf.(5), because it is $O\left(t^{-\frac{1}{2}} e^{-t / T_{1}}\right)$ instead of $O\left(t^{-\frac{3}{2}} e^{-t / T_{1}}\right)$. But one should be careful with comparing the result of theorem 2 with (5), because the given asymptotic terms are not necessarely bounds for $\left|p_{j}(t)-p_{j}(\infty)\right|, j=0,1,2$. Moreover, the coefficient in the expansion of $p_{0}(t)$ in the case $a_{1}=a_{2}<1$ vanishes as $a_{1} \rightarrow 1$, whereas that of $p_{1}(t)$ tends to infinity as $a_{1} \rightarrow 1$.

Theorem 3. When the tandem system is null-recurrent, as $t \rightarrow \infty$,

$$
\begin{gathered}
p_{0}(t)=\frac{1-a_{2}}{\sqrt{\pi t / \beta_{1}}}\left[1+O\left(\frac{1}{t}\right)\right], \quad \text { if } a_{1}=1, a_{2}<1, \\
p_{0}(t)=\frac{1-a_{1}}{\sqrt{\pi t / \beta_{2}}}\left[1+O\left(\frac{1}{t}\right)\right], \quad \text { if } a_{1}<1, a_{2}=1, \\
p_{0}(t)=O\left(\frac{1}{t}\right), \quad \text { if } a_{1}=a_{2}=1 .
\end{gathered}
$$

Proof. In the first two cases the asymptotic behaviour of $p_{0}(t)$ is determined by the singularity of $\Omega(\rho ; 0,0)$ at $\rho_{1}=0$ and $\rho_{2}=0$ respectively, so that the expansions follow from lemma 9 and (5). In the case $a_{1}=a_{2}=1$ the transform $\Omega(\rho ; 0,0)$ is given by (26) for $\rho>\rho_{0}=0$, cf.(15). The expression (26) for 
$\Omega(\rho ; 0,0)$ can be rewritten in the form $\left(a_{1}=a_{2}\right)$

$$
\begin{gathered}
{\left[\gamma^{\prime}(\rho ;-1)\right]^{-2} \frac{\beta_{1}}{2 \pi} \int_{0}^{\pi} M(\rho ; \theta) \frac{\theta d \theta}{\left|1-\gamma\left(\rho ; e^{i \theta}\right)\right|^{3}},} \\
M(\rho ; \theta):=\left|\frac{\gamma\left(\rho ; e^{i \theta}\right)}{1+e^{i \theta}}\right|^{2} \frac{1-e^{i \theta}}{1+e^{i \theta}} \frac{\gamma\left(\rho ; e^{i \theta}\right)-\gamma\left(\rho ; e^{-i \theta}\right)}{\theta\left|1-\gamma\left(\rho ; e^{i \theta}\right)\right|} .
\end{gathered}
$$

With the aid of lemma 4 it follows that as $u \rightarrow 1,|u| \leqslant 1$,

$$
\gamma\left(\rho_{0} ; u\right)=x\left(\rho_{0}\right)-[(1-u) g]^{\frac{2}{3}}+O\left(|1-u|^{\frac{4}{3}}\right),
$$

here $g$ is a constant which can be calculated from the results of the appendix, (A.6), (A.7), noting that $x^{3}\left(\rho_{0}\right)=a_{1} / a_{2}^{2}(=1)$ :

$$
\gamma_{0}^{\prime}\left(\rho_{0} ; 0\right) / g=4\left[x\left(\rho_{0}\right)\right]^{-\frac{5}{2}}=4
$$

Next we rewrite (44) as follows

$$
\begin{aligned}
& \Omega(\rho ; 0,0)=\left[\gamma_{0}{ }^{\prime}(\rho ; 0)\right]^{2} \frac{\beta_{1}}{2 \pi}\left[\int_{0}^{\pi} \frac{M(0,0) \theta d \theta}{[1-x(\rho)]^{3}+g^{2} \theta^{2}}+\right. \\
& \left.+\int_{0}^{\pi}\left\{\frac{M(\rho ; \theta)}{|1-\gamma(\rho ; \theta)|^{3}}-\frac{M(0 ; 0)}{[1-x(\rho)]^{3}+g^{2} \theta^{2}}\right\} \theta d \theta\right] .
\end{aligned}
$$

The second integral is finite for $\rho=\rho_{0}$, because it follows from (45),(46), that its integrand is $O\left(\theta^{-\frac{1}{3}}\right)$ for $\rho=\rho_{0}$ as $\theta \downarrow 0$. Hence, with (47) and $M(0,0)=\frac{1}{8} \sqrt{3}$, cf.(45),(46), and with, cf. (20), as $\rho \downarrow \rho_{0}$,

$$
x(\rho)=\frac{1}{a_{2}}\left(a_{1} a_{2}\right)^{\frac{1}{3}}-\left[\frac{1}{3} \alpha\left(\rho-\rho_{0}\right) a_{1} / a_{2}\right]^{\frac{1}{2}}+o\left(\left[\rho-\rho_{0}\right]^{\frac{1}{2}}\right),
$$

it is obtained from (48) that in the case $a_{1}=a_{2}=1$,

$$
\lim _{\rho \downarrow 0}\left[\Omega(\rho ; 0,0)-\frac{3 \sqrt{3}}{4 \pi} \beta \log \frac{1}{\rho}\right]=\text { const. }
$$

Because, as $\rho \downarrow 0$,

$$
\log \frac{1}{\rho}=\int_{1}^{\infty} \frac{1}{t} e^{-\rho t} d t+O(1)
$$

the assertion follows.

For the transient cases it is noted that the asymptotic expansion is the same as in the ergodic cases (deleting the stationary term), when the relaxation time is $T_{1}$ or $T_{2}$, cf. theorem 1 . When the relaxation time is $T_{0}$ the asymptotic expansion has to be obtained from the integral $I_{C}(\rho)$, cf.(27), which seems to be difficult because of the singularities of $\gamma(\rho ; u)$ at $\rho=\rho_{0}, u=1, \mathrm{cf}(46),(49)$. It is only stated here, that in the cases $a_{1}=a_{2}^{2}$ and $a_{2}=a_{1}^{2}$ it has been found that $p_{0}(t)=o\left(t^{-2} e^{-t / T_{j}}\right), t \rightarrow \infty, j=1,2$ respectively. The deviating behaviour in the case $a_{2}=a_{1}^{2}$ is probably related to the fact that when $a_{1}>1$ the load of the second station is $a_{2} / a_{1}$ so that $a_{2}=a_{1}^{2}$ means that the stations are equally loaded. The case $a_{1}=a_{2}^{2}$ is less clear, it seems only to have meaning for the reversed process. 


\section{Appendix on the conformal mapping}

In this appendix it will be shown how the conformal mapping $\gamma(\rho ; z)$ can be continued analytically into the region $|z|>1$. Further an explicit expression will be derived for the inverse conformal mapping $\gamma_{0}\left(\rho ; z_{2}\right)$, which makes it possible to determine the constants in the asymptotic expansions in section 7.

Because $\gamma(\rho ; u) \in Z(\rho)$ for $|u|=1$, it satisfies for $|u|=1$, cf.(17),(22),

$$
\gamma(\rho ; u) \gamma\left(\rho ; \frac{1}{u}\right)=\frac{1}{2} \frac{a_{1}}{a_{2}}\left\{A(\rho)-\sqrt{A^{2}(\rho)-\frac{4}{a_{1}}\left[\gamma(\rho ; u)+\gamma\left(\rho ; \frac{1}{u}\right)\right]},\right.
$$

or

$$
\frac{a_{2}}{a_{1}} \gamma^{2}(\rho ; u) \gamma^{2}\left(\rho ; \frac{1}{u}\right)-A(\rho) \gamma(\rho ; u) \gamma\left(\rho ; \frac{1}{u}\right)+\frac{1}{a_{2}}\left\{\gamma(\rho ; u)+\gamma\left(\rho ; \frac{1}{u}\right)\right\}=0 .
$$

Because $\gamma\left(\rho ; \frac{1}{u}\right)$ is a regular function for $|u|>1$, this relation can serve as an analytic continuation of $\gamma(\rho ; u)$ into the region $|u|>1$. It can be shown that the only singularities of $\gamma(\rho ; u)$ are two branch points on the real interval $[1, \infty]$ when $\rho \geqslant \rho_{0}$. By taking $u=\gamma_{0}(\rho ; 1)$ and $u=\gamma_{0}\left(\rho ; a_{1} / a_{2}\right)$ respectively in relation (A.2) it follows readily, cf.(3), that

$$
\gamma\left(\rho ; \frac{1}{\gamma_{0}(\rho ; 1)}\right)=\frac{a_{1}}{a_{2}} \nu_{1}(\rho), \quad \gamma\left(\rho ; \frac{1}{\gamma_{0}\left(\rho ; a_{1} / a_{2}\right)}\right)=\nu_{2}(\rho) .
$$

Next, the inverse $\gamma_{0}\left(\rho ; z_{2}\right)$ of $\gamma(\rho ; z)$ will be determined. From (1) it is clear that in the case $a_{1}<1, a_{2}<1$,

$$
\lim _{\rho \downarrow 0} \rho \Omega\left(\rho ; z_{1}, z_{2}\right)=\frac{1-a_{1}}{1-a_{1} z_{1}} \frac{1-a_{2}}{1-a_{2} z_{2}} .
$$

On the other hand, from the solution of the Riemann-Hilbert problem, cf.(29) with $z=\gamma_{0}\left(\rho ; z_{2}\right)$, it can be obtained with a similar method as in [1], theorem II.8.7, that in the case $a_{1}=a_{2}<1$,

$$
\lim _{\rho \downarrow 0} \rho \Omega\left(\rho ; 0, z_{2}\right)=\frac{1}{4} \frac{1-a_{1}}{\left[\gamma^{\prime}(0 ; 1)\right]^{2}}\left[\frac{1+\gamma_{0}\left(0 ; z_{2}\right)}{1-\gamma_{0}\left(0 ; z_{2}\right)} \frac{1-z_{2}}{z_{2}}\right]^{2} .
$$

From (A.4) and (A.5) the conformal mapping $\gamma_{0}\left(\rho ; z_{2}\right)$ can be determined in the special case $a_{1}=a_{2}<1, \rho=0$. However, the contour $Z(\rho), a_{1}=a_{2}<1, \rho=0$, can be transformed into any contour $Z(\rho)$ by means of the substitution, cf. section 3,(17),(20),

$$
\frac{2}{a_{1}}=\frac{2}{a_{2}} \rightarrow \frac{a_{1} A(\rho)}{a_{2} x^{2}(\rho)}-1, \quad z_{2} \rightarrow z_{2} / x(\rho) .
$$

Hence, by this transformation the conformal mapping $\gamma_{0}\left(\rho ; z_{2}\right)$ can be obtained for every value of $a_{1}, a_{2}$ and $\rho$. After some straightforward calculation it is found:

$$
\gamma\left(\rho ; z_{2}\right)=\frac{z_{2} h(\rho ; \phi)-\phi h\left(\rho ; z_{2}\right)}{z_{2} h(\rho ; \phi)+\phi h\left(\rho ; z_{2}\right)},
$$

here

$$
h\left(\rho ; z_{2}\right):=\left[x(\rho)-z_{2}\right]\left\{a_{1}-a_{2}^{2} z_{2} x^{2}(\rho)\right\}^{\frac{1}{2}},
$$

and $\phi:=\gamma(\rho ; 0)$ is some unspecified constant on the interval $(0, x(\rho))$. Because $x=x(\rho)$ is for real $\rho, \rho>\rho_{0}$, the smallest positive root of equation (20), and because this equation (20) has for $\operatorname{Re} \rho>\rho_{0}$ a unique root in the domain $|x|<\left(a_{1} / a_{2}^{2}\right)^{\frac{1}{3}}$ (Rouché's theorem), $x(\rho)$ can be continued as a regular 
function into the half plane $\operatorname{Re} \rho>\rho_{0}$. Therefore, also the conformal mapping $\gamma_{0}\left(\rho ; z_{2}\right)$ and its inverse can be continued into this half plane. From (A.6) and (A.7) it follows that

$$
\begin{aligned}
& z_{2}^{3}\left[\gamma_{0}^{\prime}(\rho ; 0)\right]^{2} \gamma_{0}{ }^{\prime}\left(\rho ; z_{2}\right) \frac{1-\gamma_{0}\left(\rho ; z_{2}\right)}{\left[1+\gamma_{0}\left(\rho ; z_{2}\right)\right]^{3}}= \\
& =\frac{2 h\left(\rho ; z_{2}\right)}{[h(\rho ; 0)]^{2}}\left[h\left(\rho ; z_{2}\right)-z_{2} h^{\prime}\left(\rho ; z_{2}\right)\right]=2-a_{2} z_{2} A(\rho)+\frac{a_{2}^{2}}{a_{1}} z_{2}^{3} .
\end{aligned}
$$

Note that this expression is independent of the constant $\phi$ and even of the function $x(\rho)$. Since $\gamma^{\prime}\left(\rho ; \gamma_{0}\left(\rho ; z_{2}\right)\right) \gamma_{0}{ }^{\prime}\left(\rho ; z_{2}\right)=1$ for all $z_{2}$, (A.8) can be used to determine a part of the term (33) by taking $z_{2}=1$, and similarly it leads to (35) by taking $z_{2}=a_{1} / a_{2}$.

\section{References}

[1] Blanc, J.P.C. (1982) Application of the Theory of Boundary Value Problems in the Analysis of a Queueing Model with Paired Services, Mathematical Centre Tract 153, Amsterdam.

[2] Blanc, J.P.C. (1982) Asymptotic analysis of a queueing system with a two-dimensional state space, Report BW 172/82, Mathematical Centre, Amsterdam.

[3] Cohen, J.W. (1982) The Single Server Queue, North-Holland Publ.Co., Amsterdam, 2 nd ed.

[4] Cohen, J.W. \& Boxma, O.J. (1983) Boundary Value Problems in Queueing System Analysis, North-Holland Publ.Co., Amsterdam.

[5] Doorn, E.A. van (1981) Stochastic Monotonicity and Queueing Applications of Birth-Death Processess, Springer Verl., New York.

[6] Fayolle, G. \& Iasnogorodski, R. (1979) Two coupled processors: the reduction to a RiemannHilbert problem, Z-Wahrscheinlichtkeitstheor. Verw. Gebiete 47, pp. 325-351.

[7] Fayolle, G., King, P.J.B. \& Mitrani, I. (1982) The solution of certain two-dimensional Markov models, Adv.Appl. Probab. 14, pp. 295-308.

[8] Harrison, P.G. (1981) Transient behaviour of queueing networks, J. Appl. Probab 18, pp. 482490.

[9] Jackson, R.R.P. (1954) Queueing systems with phase-type service, Oper. Res. Quart. 5, pp. 109120.

[10] Kobayashi, H. (1974) Application of the diffusion approximation to queueing networks II : nonequilibrium distributions and applications to computer modelling, J.A.C.M. 21, pp. 459-469.

[11] Muschelischwili, N.I. (1965) Singuläre Integralgleichungen, Akademie-Verlag, Berlin.

[12] Tsuji, M. (1959) Potential Theory in Modern Function Theory, Maruzen Co., Tokyo.

[13] Whitt, W. (1983) Untold horrors of the waiting room: What the equilibrium distribution will never tell about the queue-length process, Man. Sci. 29, pp. 395-408.

[14] Wishart, D.M.G. (1966) Discussion on Kingman's paper, J. Roy. Statist. Soc. Ser. B 28, pp. 442,443 . 